\title{
Analysis of Scaling Law and Figure of Merit of Fiber-Based Biosensor
}

\author{
Jui-Teng Lin, ${ }^{1}$ Da-Chuan Cheng, ${ }^{2}$ Minshan Jiang, ${ }^{3}$ Yueh-Sheng Chiang, ${ }^{4}$ and Hsia-Wei Liu ${ }^{5}$ \\ ${ }^{1}$ New Vision Inc., Banciao, New Taipei City, Taiwan \\ ${ }^{2}$ Department of Biomedical Imaging and Radiological Science, China Medical University, Taichung, Taiwan \\ ${ }^{3}$ Optical Electronic Information and Computer, Engineering College, University of Shanghai for Science and Technology, \\ Shanghai, Taiwan \\ ${ }^{4}$ The Graduate Institute of Applied Science and Engineering, Fun Jen Catholic University, New Taipei City, Taiwan \\ ${ }^{5}$ Department of Life Science, College of Science and Engineering, Fun Jen Catholic University, New Taipei City, Taiwan
}

Correspondence should be addressed to Hsia-Wei Liu,079336@mail.fju.edu.tw

Received 28 March 2012; Revised 14 May 2012; Accepted 14 May 2012

Academic Editor: Christian Brosseau

Copyright ( $\odot 2012$ Jui-Teng Lin et al. This is an open access article distributed under the Creative Commons Attribution License, which permits unrestricted use, distribution, and reproduction in any medium, provided the original work is properly cited.

This paper presents a normalized transmitted signal (NTS) of a fiber-based sensor using gold nanorods as the plasmon excitation medium of the evanescent wave. The NTS and the refractive index (RI) sensitivity is calculated as a function of the gold aspect ratio $(R)$, the RI of the sensing medium, and a scaling parameter given by the ratio of the fiber length and its diameter. Finally, the optimal value of gold aspect ratio is calculated to be $R=(3.0-4.0)$ for maximum figure of merits (FOMs) defined by the ratio of the refractive index sensitivity and the full width at half maximum. The scaling laws and the FOM presented in this paper may serve as the guidelines for optimal designs in fiber-based nanosensors.

\section{Introduction}

The use of metal nanoparticles in biomedicine includes bioimaging, biosensing, drug delivery, and diagnostics therapeutics of cancer cell [1-14]. Surface plasmon resonances (SPRs) of various shapes of nanoparticles have been explored including shapes in sphere, rod, box, and core shell [48]. By changing the shapes of nanoparticles from spheres to nanorods, the absorption and scattering peaks change from visible to the near infrared (NIR) region and offer the advantages of larger absorption/scattering cross sections and much deeper penetration depth in tissues [4-9]. The resonance wavelength of SPR is highly sensitive to the local environment of the nanoparticles, such as, the refractive index (RI) and temperature of the surrounding solvent, and the binding agents to the functionalized nanoparticles [4$10]$.

Conventional SPR sensors are difficult to be integrated in a large-scale array format. The recently developed fiberbased sensors [11-17] benefit is easy to fabricate and requires only a fiber-deliver of the lights and spectrometer for detection. Sensors operate in the NIR region is potentially useful for detecting low concentration of analytes functionalized nanoparticles due to its much higher refractive index sensitivity. For example, the sensitivity is about 200$400(\mathrm{~nm} / \mathrm{RIU})$ in gold nanorods (GNRs) comparing about $50-80(\mathrm{~nm} / \mathrm{RIU})$ in gold nanosphere $[7,9,10,18]$.

The performance of a fiber-based sensor is characterized by not only the refractive index sensitivity function $(M)$, but more importantly, the figure of merit (FOM) defined by the ratio of FOM $=M / F W H M$, where FWHM is the full width at half maximum of the absorptivity of the nanoparticles coated on the fiber core surface.

This study presents a normalized transmitted signal (NTS) of a fiber-based sensor using gold nanorods (GNRs) as the plasmon excitation medium of the evanescent wave. The NTS and the refractive index (RI) sensitivity will be calculated as a function of the GNR aspect ratio $(R)$ and the RI of the sensing medium. A scaling law based on the ratio of the fiber length $(L)$ and diameter $(d)$ is presented. We will calculate the optimal value of GNR aspect ratio for maximum FOM. Analytic formula and fit-equation for 
the resonance wavelength, NTS, and $M$ will be derived and compared with the numerical results. The scaling laws and the FOM for a fiber-sensor presented in this paper are new features which have not been explored theoretically or experimentally by prior arts. Therefore, they may serve as the guideline for optimal designs in fiber-based biosensors.

\section{Theory}

The basic structure of a fiber-based sensor is shown in Figure 1, where the standing wave becomes evanescent wave when it propagates in the bared-fiber portion which is functionalized by gold nanorod (GNR) layer as the absorbing medium. In this paper, we consider the situation that the GNRs are very thick layer (or low concentration) and will be uniformly surrounded by the sensing solution which affects the nanogold absorption peak shift by its index $(n)$ changes.

The transmitted light intensity $(I)$ is related to the input intensity $\left(I_{0}\right)$ of the fiber-based nanosensor as follows [1517]:

$$
\frac{I}{I_{0}}=\left(\frac{N_{2}}{N_{1}}\right)^{2} 10^{-A F c L},
$$

where $F$ is the power fraction of the evanescent wave in the GNR-layer deposited portion of the fiber having a length of $L$, given by [15]:

$$
F=\frac{0.0006 \lambda}{\left(d N_{2}\right)}
$$

in which the unit of the light wavelength $(\lambda)$ is $\mathrm{nm}$ and fiber core diameter $(d)$ is um. The numerical aperture $N_{1}$ and $N_{2}$ are defined by the refractive index (RI) of the fiber core $\left(n_{1}\right)$, cladding $\left(n_{2}\right)$ and the sensing medium $(n)$ by [16]:

$$
N_{1}=\sqrt{n_{1}^{2}-n_{2}^{2}}, \quad N_{2}=\sqrt{n_{1}^{2}-n^{2}},
$$

$A$ is the effective absorptivity of the absorbing GNR having a molar concentration $c$ is given by the wavelength-dependent equation based on Gans theory [18, 19]:

$$
A(\lambda)=\left(\frac{2.094 n^{3}}{\lambda}\right)\left(\frac{\varepsilon^{\prime} / P^{2}}{\varepsilon^{\prime 2}+\Delta^{2}}\right)
$$

where

$$
\begin{gathered}
\Delta=\frac{\varepsilon+n^{2}(1-P)}{P}, \\
P=\left(\frac{1-e^{2}}{e^{2}}\right)\left[\frac{1}{2 e} \ln \left(\frac{1+e}{1-e}\right)-1\right], \\
e=\sqrt{1-R^{-2}} .
\end{gathered}
$$

The real $(\varepsilon)$ and imaginary part $\left(\varepsilon^{\prime}\right)$ of gold dielectric function, reported by Johnson and Christy [20], may be fit to the follow nonlinear equation $[21,22]$,

$$
\varepsilon=14.49-0.01375 \lambda-0.000043 \lambda^{2},
$$

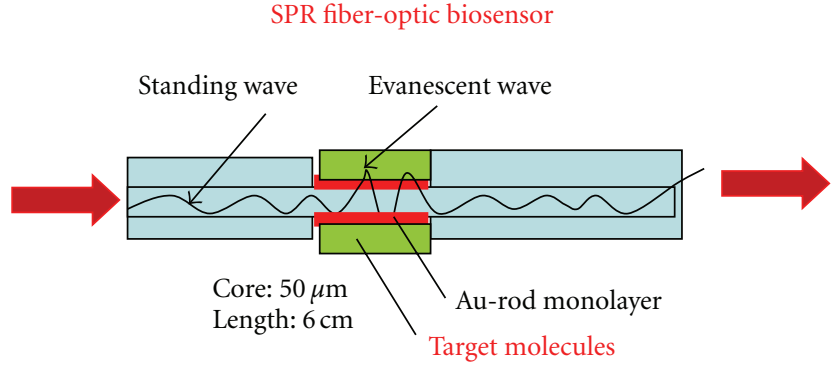

FIGURE 1: A fiber-based sensor showing the standing and evanescent wave. The bared-fiber portion is functionalized by nanogold layer for a length $L$ and core diameter $d$.

for all range of aspect ration $R=(1.0-10)$ and the imaginary part:

$$
\varepsilon^{\prime}=0.00005 \lambda^{2}-0.071 \lambda+25.65,
$$

for $R=1.0$ to 3.0 and

$$
\varepsilon^{\prime}=0.000011 \lambda^{2}-0.0127 \lambda+4.6,
$$

for $R>3.0$.

At resonance condition given by $\Delta=\varepsilon+n^{2}(1-P) / P=0$ for maximum $A(\lambda)$ of $(4 \mathrm{a})$, the transmitted light intensity $(I)$ is given by:

$$
\begin{gathered}
I\left(\lambda^{*}\right)=I_{0}\left(\frac{N_{2}}{N_{1}}\right)^{2} 10^{-1.26 G^{*}}, \\
G^{*}=\left(\frac{n^{3}}{\varepsilon^{\prime}\left(\lambda^{*}\right)}\right)\left(\frac{0.001 c L}{d N_{2}}\right)(3.75 R-1.95)^{2},
\end{gathered}
$$

where we have used the linear approximation of $1 / P=$ $3.75 R-1.95$ in $(4 \mathrm{c})[6,18,21]$.

The resonance wavelength $\left(\lambda^{*}\right)$ is related to the GNR aspect ratio $(R)$ and the sensing medium $\mathrm{RI}(n)$ by an analytic equation found by curve fitting to the numerical calculation based on Gans theory $[19,21]$.

$$
\lambda^{*}=395+86.5 n R-28 R+3.5 n^{2} R+5 n^{3} .
$$

When we use water as the reference medium, the resonance effective absorptivity $\left(A_{1}\right)$ should be also defined by the resonance wavelength $\left(\lambda^{*}\right)$ at the $\mathrm{RI}$ of water $(n=1.33)$, such that (7) becomes only $R$-dependent as follows:

$$
\lambda^{*}(n=1.33)=406.9+93.2 R,
$$

which is a linearly increasing function of $R$. In comparison, for a fixed GNR aspect ratio at $R=4.0$ (for peak absorption around $810 \mathrm{~nm})$, the resonance wavelength $\left(\lambda^{*}\right)$ has a nonlinear $n$-dependent as follows:

$$
\lambda^{*}(R=4.0)=283+346 n+14 n^{2}+5 n^{3} .
$$

Using the resonance-transmitted intensity in water (with $\mathrm{RI}=1.33$ ) as the reference, we define a normalized, offresonance, transmitted signal (NTS) of the sensing medium (with $\mathrm{RI}=n) S=I\left(\lambda^{*}, n\right) / I\left(\lambda^{*}, n=1.33\right)$ is given by:

$$
S=\left(\frac{N_{22}}{N_{21}}\right)^{2} 10^{\left(A_{1} F_{1}-A_{2} F_{2}\right) L}
$$


where we further define the numerical aperture in water (reference) and sensing medium,

$$
N_{21}=\sqrt{n_{1}^{2}-1.33^{2}}, \quad N_{22}=\sqrt{n_{1}^{2}-n^{2}} .
$$

The power fractions of the evanescent wave are valued at the resonance wavelength $\left(\lambda^{*}\right)$, that are,

$$
F_{1}=\frac{0.0006 \lambda^{*}}{\left(d N_{21}\right)}, \quad F_{2}=\frac{0.0006 \lambda^{*}}{\left(d N_{22}\right)} .
$$

The normalized effective absorptivity (per molar), $A j(j=$ $1,2)=A / c$, is valued at the resonance wavelength $\left(\lambda^{*}\right)$ given by:

$$
\begin{gathered}
A_{1}=\left(\frac{2.094}{\lambda^{*}}\right)\left(\frac{1.33^{3}}{P^{2} \varepsilon^{\prime}}\right), \\
A_{2}=\left(\frac{2.094 n^{3}}{\lambda^{*}}\right)\left(\frac{\varepsilon^{\prime} / P^{2}}{\varepsilon^{\prime 2}+\left[\left(n^{2}-1.33^{2}\right)(1-P) / P\right]^{2}}\right) .
\end{gathered}
$$

We further define the intensity sensitivity $(M)$ of the normalized transmitted signal (NTS) by taking the derivative of the NTS with respect to the sensing medium RI $(n)$ as follows:

$$
M=-\frac{d S}{d n} .
$$

In comparison, the refractive-index-sensitivity is defined by [18]:

$$
M^{\prime}=\frac{d \lambda^{*}}{d n}
$$

which may be analytically found from the derivative of (7)

$$
M^{\prime}=86.5 R+7 n R+15 n^{2} .
$$

The goal of this study is to calculate these sensitivity functions and analyze the roles of the fiber parameters (core size, fiber length), the RI of the medium, and aspect ratio of the GNR. Finally, we will calculate the figure of merit (FOM) of the fiber sensor, defined by the ratio of

$$
\mathrm{FOM}=\frac{M}{\mathrm{FWHM}},
$$

where FWHM is the full width (broadening) at half maximum of the NTS at a given light wavelength and GNRs aspect ratio $(R)$.

\section{Results and Discussion}

3.1. The Transmitted Signal. We will use a silica fiber with core RI $n_{1}=1.46$ in the all the following calculations. We first show in Figure 2 the profiles of the transmitted intensity based on (1) for a reference medium of water (with $\mathrm{RI}=1.33$ ), where the position of the absorption peak (or transmission dips) is red shifted for larger $n$ as shown by (8a). Furthermore, the peak intensity $I\left(\lambda^{*}\right)$ given by $(6)$ is an increasing function of $R$. The profiles for fixed $R$ but vary the sensing medium RI are shown in Figures 3 and 4. Again, the profile peaks red shifted with smaller intensity $I\left(\lambda^{*}\right)$ for higher RI and the peak heights are higher for $R=6.0$ than $R=4.0$ for a fixed sensing RI $(n)$. These features are analytically expressed by (6), (8a), and (8b).

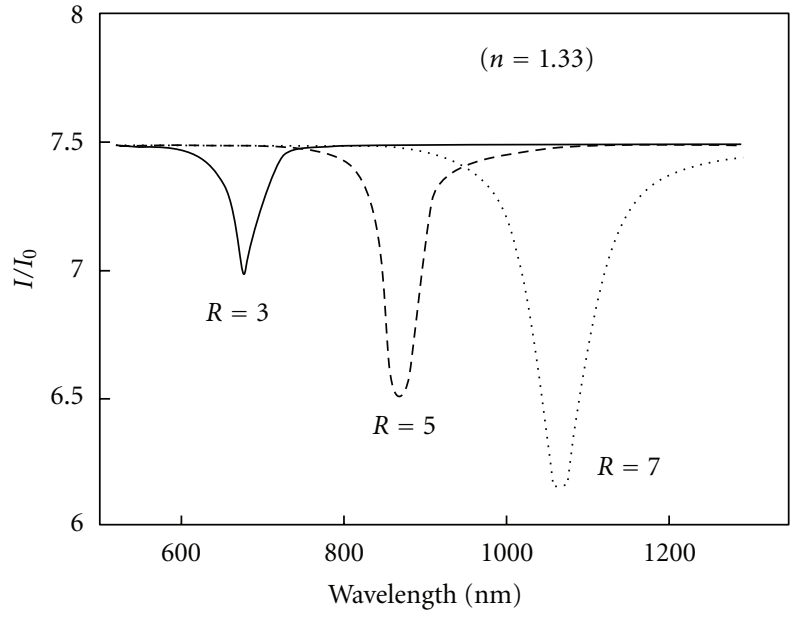

FIGURE 2: The transmitted signal profiles of reference water medium (with $\mathrm{RI}=1.33$ ) for various GNRs aspect $\operatorname{ratios}(R)$.

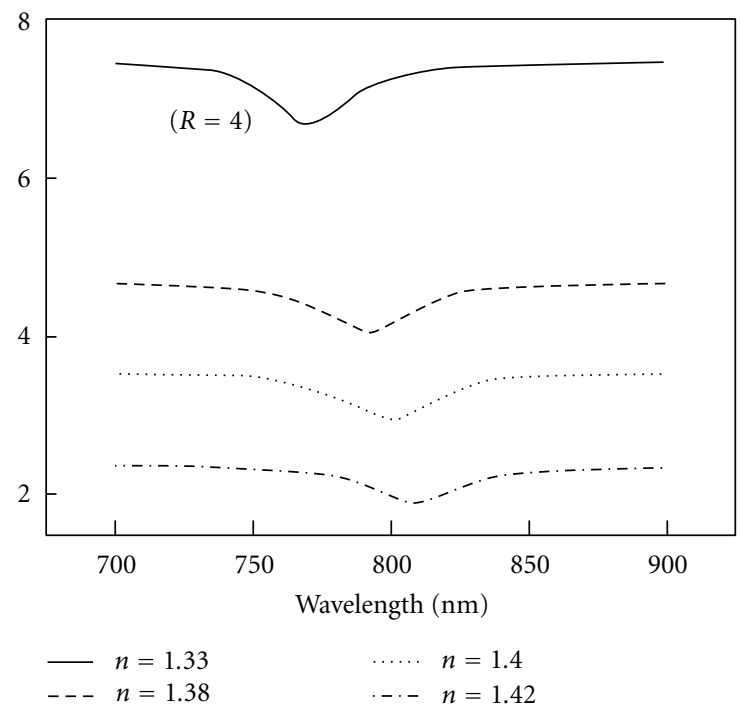

Figure 3: The transmitted signal profiles, for $R=4.0$.

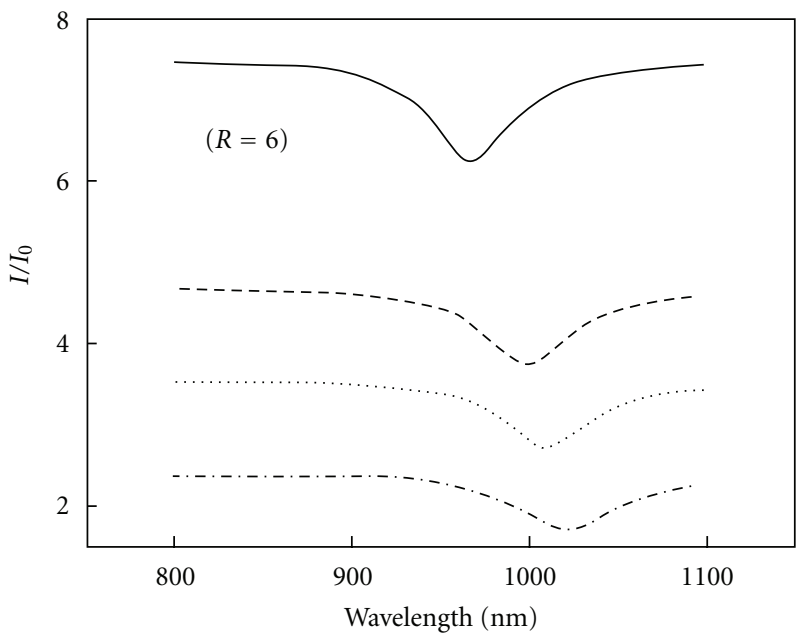

FIGURE 4: The transmitted signal profiles, for $R=6.0$. 


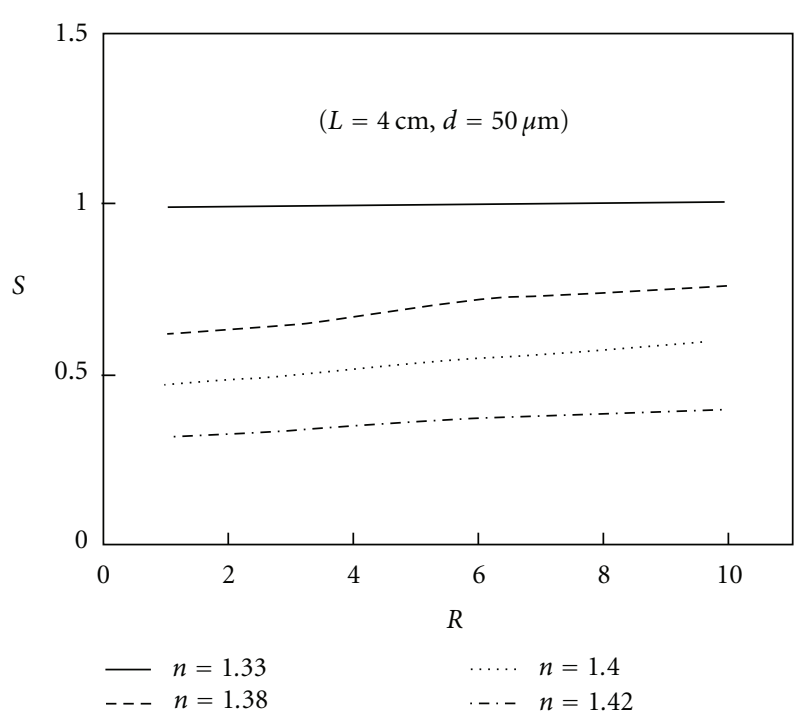

FIGURE 5: The normalized transmitted signal (NTS) versus GNRs aspect ratio $(R)$ at various sensing medium RI, for a fixed fiber length $L=4.0 \mathrm{~cm}$ and diameter $d=50 \mu \mathrm{m}$.

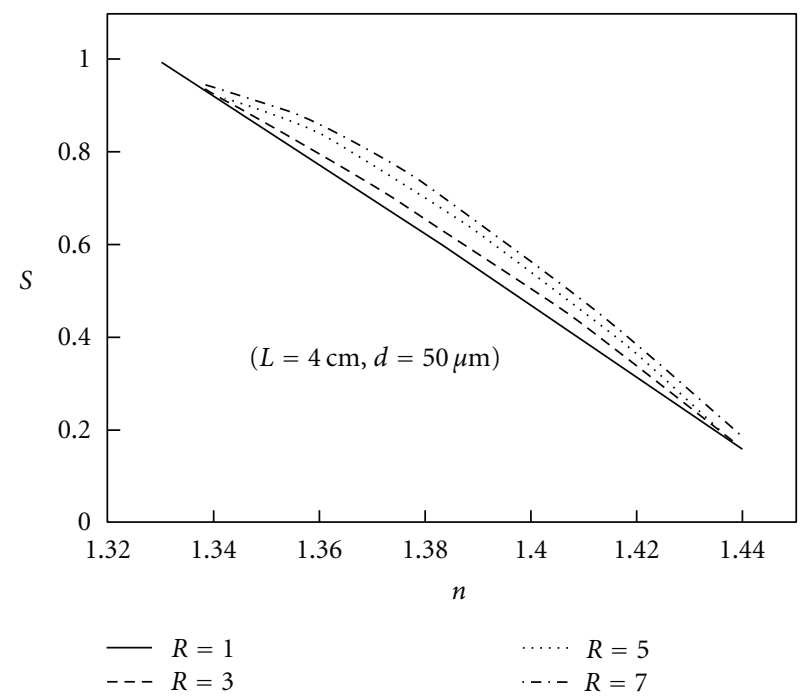

FIGURE 6: The NTS versus sensing medium RI ( $n$ ) for various ratio $(R)$.

3.2. The Normalized Transmitted Signal (NTS). In general, the normalized transmitted signal (NTS) is a function of various parameters $\left(d, L, R, n, n_{1}, n_{2}\right)$. We shall note that the NTS is independent to the fiber numerical aperture $N_{1}$, due to its normalization. In addition, the 1.33 (water RI) of (12) will be replaced by 1.00 if air is used as the reference signal. For a given aspect ratio of GNRs, we choose the associate light (laser, LED or other narrowband source) wavelength to match the resonance wavelength defined by $(8 \mathrm{a})$ and $(8 \mathrm{~b})$.

3.3. The Scaling Law. We shall now analyze the roles of various parameters on the NTS. By our definition of $S$ in (9), the NTS is a normalized, off-resonance, transmitted signal of

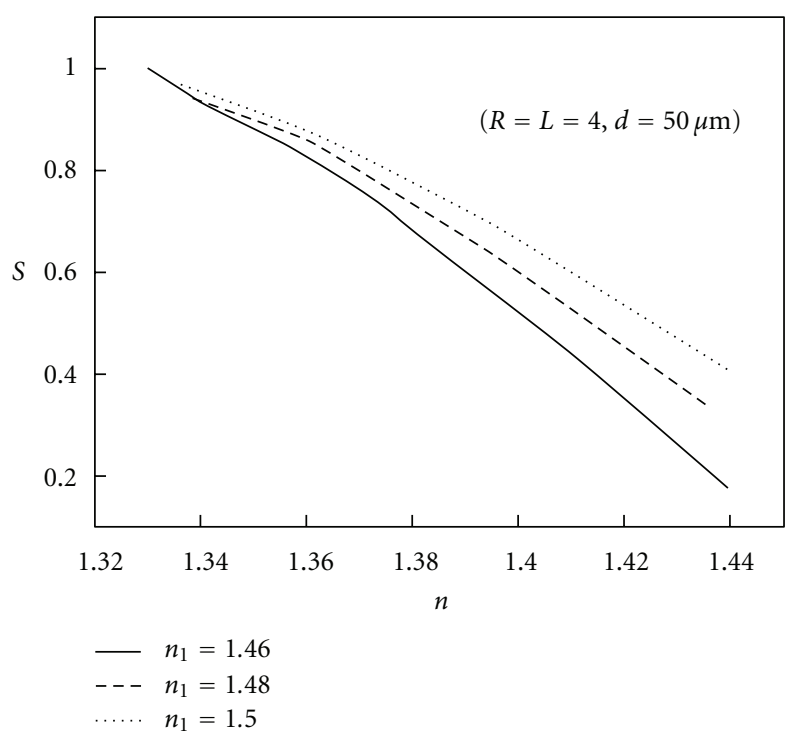

FIgURE 7: The NTS versus GNRs aspect ratio $(R)$ at various sensing medium RI, at various core RI, for fixed $R=4, L=4.0 \mathrm{~cm}$, and $d=$ $50 \mu \mathrm{m}$.

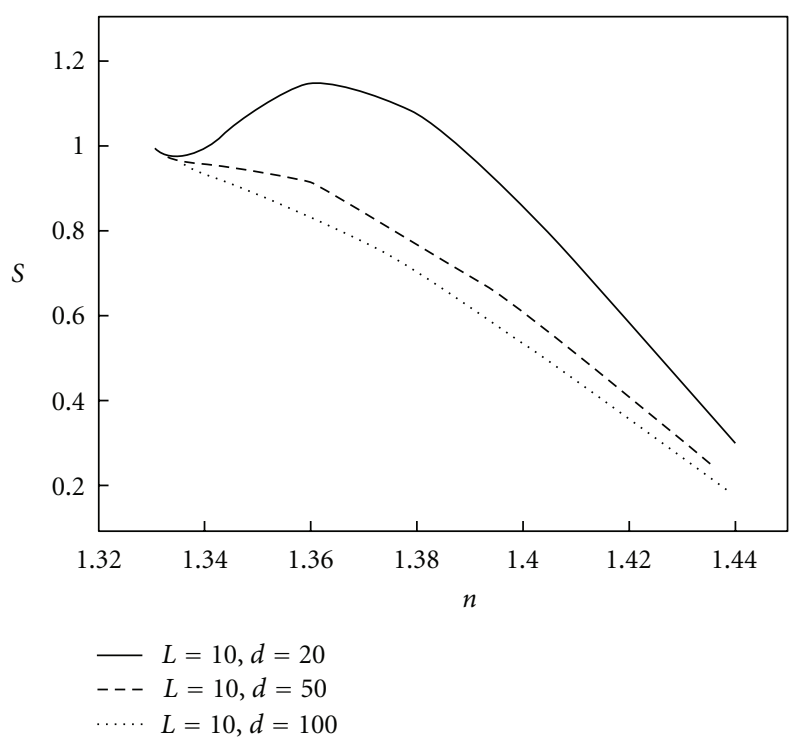

FIGURE 8: The NTS versus sensing medium RI $(n)$ for various ratio of $(L / d)$ shows higher signal for smaller core diameter.

the sensing medium. Therefore, it is expected to be a strongly decreasing function of the medium RI $(n)$ when $n$ is larger than the preset resonance RI of water at 1.33. Furthermore, the NTS should be a weakly dependent function of $R$ because of the fact that the reference and sensing medium are sharing the same GNR conditions. The in-sensitive or large tolerance feature of NTS with respect to the GNR aspect ratio offers a great advantage for sensor design. Above-predicted features are shown in Figures 5 and 6 for a given fiber length $L=$ $4.0 \mathrm{~cm}$ and core diameter $d=50 \mu \mathrm{m}$. 


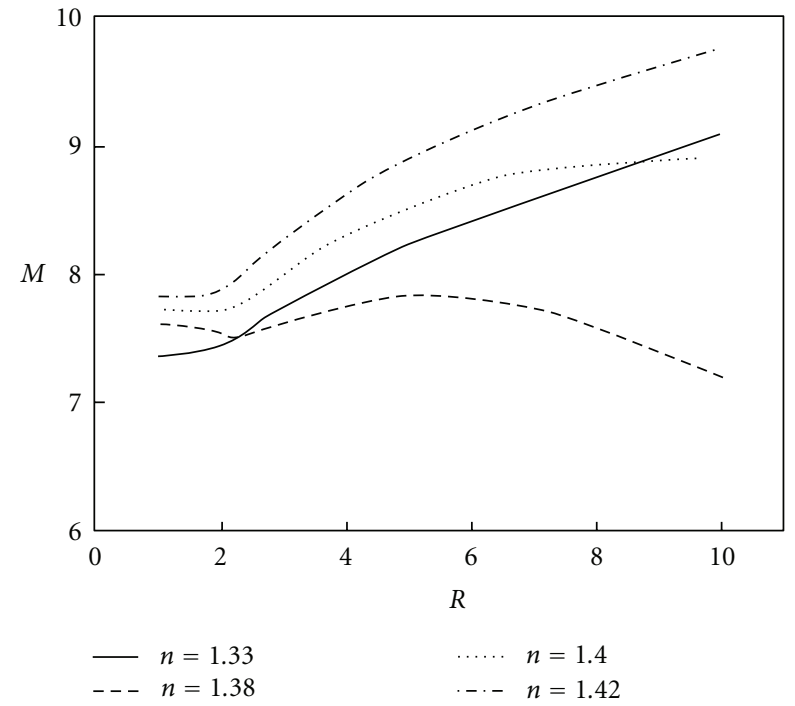

FIGURE 9: The sensitivity function $(M)$ versus medium RI ( $n$ ) associates to the slope of curves in Figure 6.

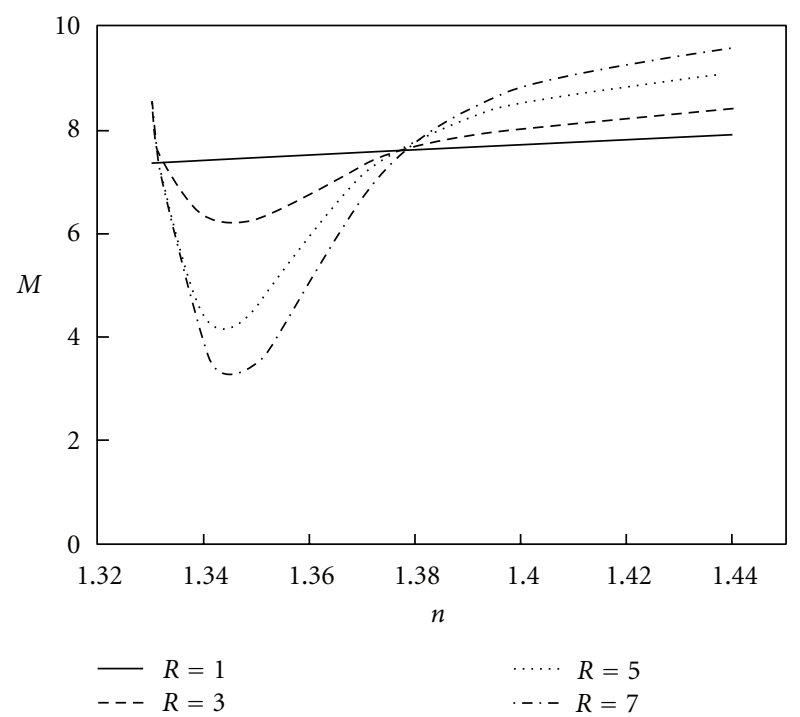

FIgURe 10: The sensitivity function $(M)$ versus $n$ for various $R$ associates to the slope of curves in Figure 6.

Figure 7 shows that the off-resonance NTS is a strong decreasing function of medium RI $(n)$ but an increasing function of the core RI $\left(n_{1}\right)$. These features may be realized by (10)-(12), where larger $n$ and smaller $n_{1}$ give smaller numerical aperture $\left(N_{22}\right)$ of the medium. We should also note that the NTS is proportional to the ratio of $(L / d)$ rather than $L$ or $d$ alone. Therefore, for a given $L$, smaller fiber core provides a stronger NTS. This scaling law defined by $(L / d)$ is demonstrated in Figure 8. We also confirm numerically (not shown in Figure 8) that same NTS is obtained for various sets of $(L, d)=(10,100),(5,50)$, and $(2.5,25)$, which have the same ratio of $(L / d)=0.1$. This scaling law has been also found

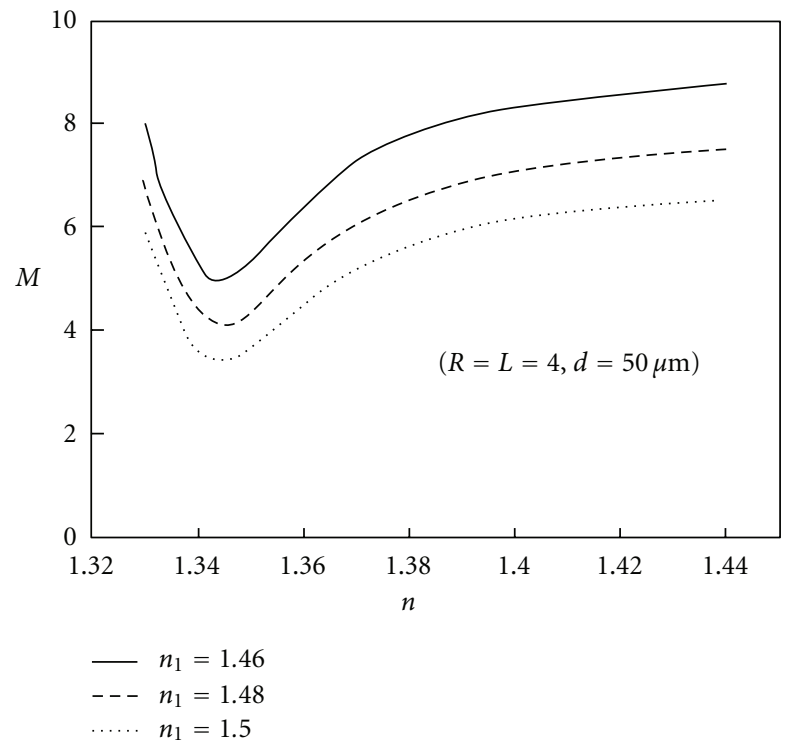

FIgURE 11: The sensitivity function $(M)$ versus $n$ associates to the slope of curves in Figure 7.

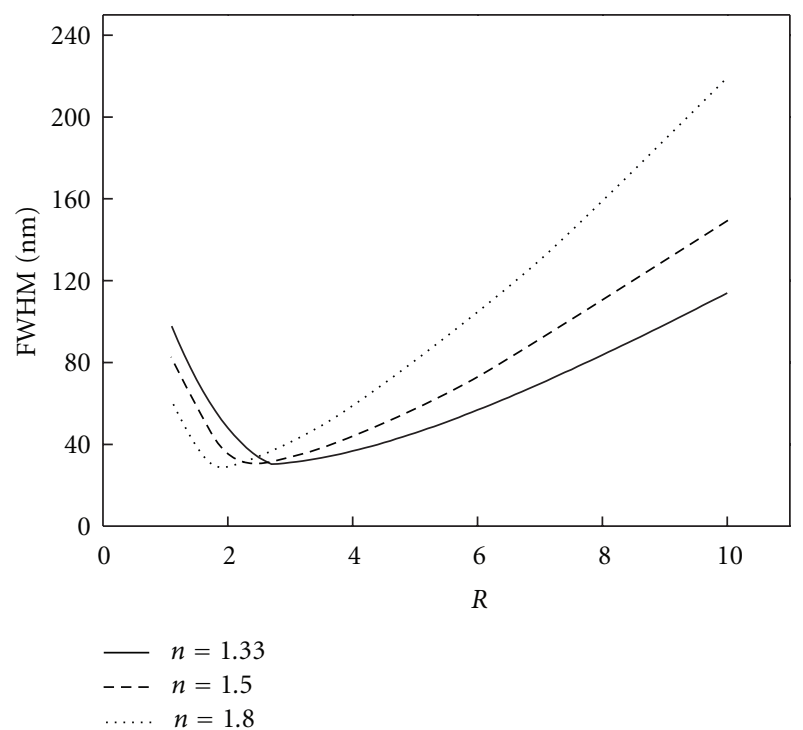

FIGURE 12: Calculated full width at half maximum (FWHM) versus aspect ratio $(R)$ of gold nanorods solution for medium RI of 1.33 (solid), 1.5 (dashed), and 1.8 (dotted) [21].

in nanoshells which has a universal parameter defined by the ratio of the shell thickness and the core diameter $[5,20]$.

3.4. The Sensitivity Function (M). We shall now present the numerical results of the sensitivity function $(M)$ defined by (13). Figures 9-12 are produced from the slopes of Figure 5-8. The important features based on these Figures may be summarized as follows: (i) $M=(7-9) \mathrm{nm}^{-1}$ is an increasing function of $R$ (see Figure 9); (ii) $M=(3-9) \mathrm{nm}^{-1}$ an increasing function for $n>1.35$ and for $L / d=0.08$ (see Figures 10-11); (iii) $M=(-10$ to 15$) \mathrm{nm}^{-1}$ an increasing 


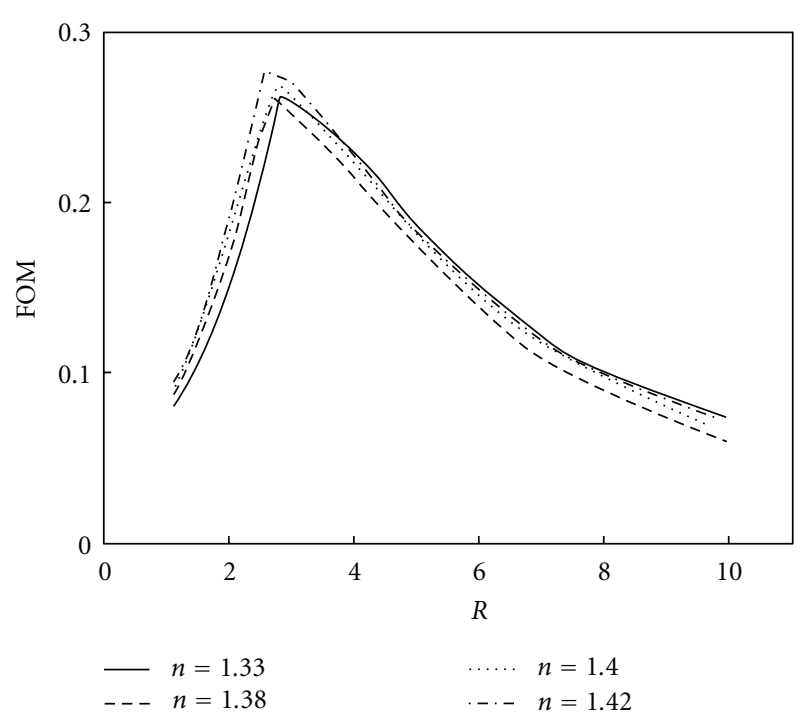

FIGURE 13: Calculated figure of merit (FOM) for fiber-based sensor defined by $\mathrm{FOM}=M / \mathrm{FWHM}$ and $M=-d S / d n$ defined by (13).

function of $R$ and ( $L / d$ ) (see Figure 12); (iv) $M$ is a nonlinear increasing function of $n$.

3.5. The Figure of Merit (FOM). The performance of a fiberbased sensor is given by not only the sensitivity functions ( $M$ and $M^{\prime}$ ) but more importantly the figure of merit (FOM) as defined earlier as FOM $=M / F W H M$ for the fiber sensor "intensity". In comparison, we may also define $\mathrm{FOM}^{\prime}=$ $M^{\prime}$ /FWHM for the GNR figure of merit for the resonance wavelength red shift, where FWHM is the full width at half maximum of the absorptivity, (12) by varying the medium RI (n) which may also be converted to the associate aspect ration $(R)$ by (7). The numerical curves for FWHM are shown in Figure 12 for gold nanorods. The sensitivity of gold nanorods defined by $M^{\prime}=d \lambda^{*} / d n$ gives the "spectrum" red shift per unit change of the medium RI. In the fiber-based device, the sensitivity is defined differently as $M=-d S / d n$, which provides the change of NTS "intensity" per unit change of the medium RI. Typical values of $M^{\prime}$ are about (100400) $\mathrm{nm} / \mathrm{RIU}$ [23] comparing to $M=(2-15) \mathrm{nm}^{-1}$.

In the experiments, one may measure both the NTS intensity change and spectrum red shift in identifying the unknown RI of the sensing medium. Given the measured values of $M$ and $M^{\prime}$ based on the RI of the reference (water $\mathrm{RI}=1.33)$, the unknown sensing medium $\mathrm{RI}(n)$ is then given by: $n=1.33-(d S / M)$ or $n=1.33+\left(d \lambda^{*} / M^{\prime}\right)$ in the linear regimes. However, the strong deviation from linearity of $\lambda^{*}$ (see (7)) and $S$ (as shown in Figures 5-8) leads to the $n$ dependence of both $M$ and $M^{\prime}$. Therefore, the linear relationship should apply only to a very small medium RI ranges, say less than 0.1 .

As shown in Figure 13, the maximum figure of merit occurs for optimal value of $R$, about 3.0 for fiber-sensortransmitted signal. In comparison, shown by Figure 14, the optimal $R=$ (3.0-4.0) for the resonance wavelength red shift in nanogoldrod solution [21]. This is an important

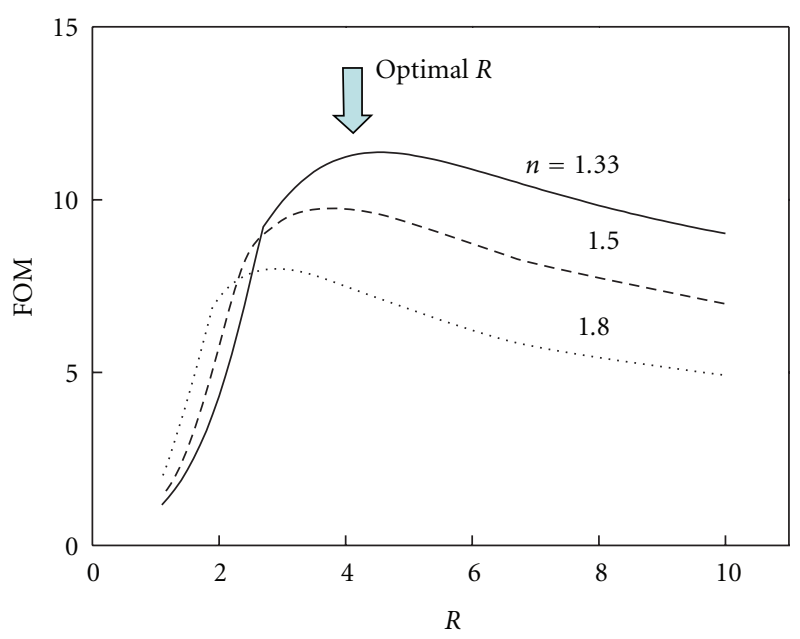

FIGURE 14: Calculated figure of merit $\left(\mathrm{FOM}^{\prime}\right)$ for gold nanorods solution [21] defined by $\mathrm{FOM}^{\prime}=M^{\prime} / \mathrm{FWHM}$, with $M^{\prime}$ defined by (14a) and (14b).

design aspect that the near IR lights (lasers or LED), with wavelength of 750-900 nm for $R=(3.5-4.2)$, match not only the optimal FOM and FOM' conditions but also provide much deeper tissue penetration than that of visible lights having wavelength $500-700 \mathrm{~nm}$ and $R=1.0-3.0$.

\section{Conclusion}

We have derived analytic formulas for the transmitted intensity in a fiber-based sensor using gold nanorods as the surface plasmon resonance medium. This study demonstrates many important design aspects based on the roles of various fiber and GNR parameters on the normalized transmitted signal (NTS) which is a strongly decreasing function of the medium RI $(n)$ and sensing numerical aperture but a weakly dependent function of the GNR aspect ratio $(R)$. The NST is characterized by the scaling parameter $(L / d)$, therefore achieving the same sensitivity, one should use a longer fiber length (the GNRS deposited portion) for a larger core diameter and vice versa. Finally, optimal figures of merits (FOM and $\mathrm{FOM}^{\prime}$ ) are found to be around $R=(3.0-4.0)$. The scaling laws and the FOM presented in this paper may serve as the guidelines for optimal designs in fiber-based sensors.

\section{Acknowledgments}

This work is partially supported by the National Science Council of Taiwan (NSC 100-2221-E-030-001). The authors thank the numerical drawings by Yu-lin Hong, a formal student of J. T. Lin at NTU, and portion of the results were published in his M. S.thesis (2010).

\section{References}

[1] P. C. Chen, S. Mwakwari, and A. K. Oyelere, "Gold nanoparticles-from nanomedicine to nanosensing," Nanotechnology, Science and Applications, vol. 1, pp. 45-66, 2008. 
[2] L. Tong, Q. Wei, A. Wei, and J.-X. Cheng, "Gold nanorods as contrast agents for biological imaging: optical properties, surface conjugation and photothermal effects," Photochemistry and Photobiology, vol. 85, no. 1, pp. 21-32, 2009.

[3] J. L. West and N. J. Halas, "Engineered nanomaterials for biophotonics applications: improving sensing, imaging, and therapeutics," Annual Review of Biomedical Engineering, vol. 5, pp. 285-292, 2003.

[4] P. K. Jain, K. S. Lee, I. H. El-Sayed, and M. A. El-Sayed, "Calculated absorption and scattering properties of gold nanoparticles of different size, shape, and composition: applications in biological imaging and biomedicine," Journal of Physical Chemistry B, vol. 110, no. 14, pp. 7238-7248, 2006.

[5] P. K. Jain and M. A. El-Sayed, "Surface plasmon resonance sensitivity of metal nanostructures: physical basis and universal scaling in metal nanoshells," Journal of Physical Chemistry C, vol. 111, no. 47, pp. 17451-17454, 2007.

[6] K. Ueno, S. Juodkazis, M. Mino, V. Mizeikis, and H. Misawa, "Spectral sensitivity of uniform arrays of gold nanorods to dielectric environment," Journal of Physical Chemistry C, vol. 111, no. 11, pp. 4180-4184, 2007.

[7] M. Cao, M. Wang, and N. Gu, "Optimized surface plasmon resonance sensitivity of gold nanoboxes for sensing applications," Journal of Physical Chemistry C, vol. 113, no. 4, pp. 1217-1221, 2009.

[8] H. Chen, X. Kou, Z. Yang, W. Ni, and J. Wang, "Shape- and size-dependent refractive index sensitivity of gold nanoparticles," Langmuir, vol. 24, no. 10, pp. 5233-5237, 2008.

[9] W. Ni, H. Chen, X. Kou, M. H. Yeung, and J. Wang, "Optical fiber-excited surface plasmon resonance spectroscopy of single and ensemble gold nanorods," Journal of Physical Chemistry C, vol. 112, no. 22, pp. 8105-8109, 2008.

[10] C. D. Chen, S. F. Cheng, L. K. Chau, and C. R. C. Wang, "Sensing capability of the localized surface plasmon resonance of gold nanorods," Biosensors and Bioelectronics, vol. 22, no. 6, pp. 926-932, 2007.

[11] C. Yu and J. Irudayara, "A multiplex biosensor using gold nanorods," Analytical Chemistry, vol. 79, pp. 572-579, 2007.

[12] R. C. Jorgenson and S. S. Yee, "A fiber-optic chemical sensor based on surface plasmon resonance," Sensors and Actuators B, vol. 12, no. 3, pp. 213-220, 1993.

[13] A. K. Sharma, R. Jha, H. S. Pattanaik, and G. J. Mohr, "Design considerations for surface plasmon resonance-based fiberoptic detection of human blood group," Journal of Biomedical Optics, vol. 14, no. 6, Article ID 064041, 2009.

[14] D. Monzón-Hernández, J. Villatoro, and D. Luna-Moreno, "Miniature optical fiber refractometer using cladded multimode tapered fiber tips," Sensors and Actuators B, vol. 110, no. 1, pp. 36-40, 2005.

[15] D. Gloge, "Weakly guiding fibers," Applied Optics, vol. 10, pp. 2252-2258, 1971.

[16] L. C. Bobbb, H. D. Krumboldt, and J. P. Davis, in Proceedings of the SPIE, vol. 990, pp. 164-169, 1988.

[17] J. P. Conzen, J. Burck, and H. J. Ache, "Characterization of a fiber-optic evanescent wave absorbance sensor for nonpolar organic compounds," Applied Spectroscopy, vol. 47, pp. 753$763,1993$.

[18] S. Link and M. A. El-Sayed, "Erratum: Simulation of the optical absorption spectra of gold nanorods as a function of their aspect ratio and the effect of the medium dielectric constant," Journal of Physical Chemistry B, vol. 109, no. 20, pp. 10531-10532, 2005.

[19] C. P. Bohren and D. R. Huffman, Absorption and Scattering of Light by Small Particles, Wiley, New York, NY, USA, 1983.
[20] P. B. Johnson and R. W. Christy, "Optical constants of the noble metals," Physical Review B, vol. 6, no. 12, pp. 4370-4379, 1972.

[21] J. T. Lin and Y. L. Hong, in Proceedings of the SPIE, vol. 7574, pp. 1-5, 2010.

[22] J.-T. Lin, "Modeling the scaling law of surface plasmon resonance in gold spherical nanoshells," Journal of Nanophotonics, vol. 4, no. 1, Article ID 049507, 2010.

[23] M. M. Miller and A. A. Lazarides, "Sensitivity of metal nanoparticle surface plasmon resonance to the dielectric environment," Journal of Physical Chemistry B, vol. 109, no. 46, pp. 21556-21565, 2005. 

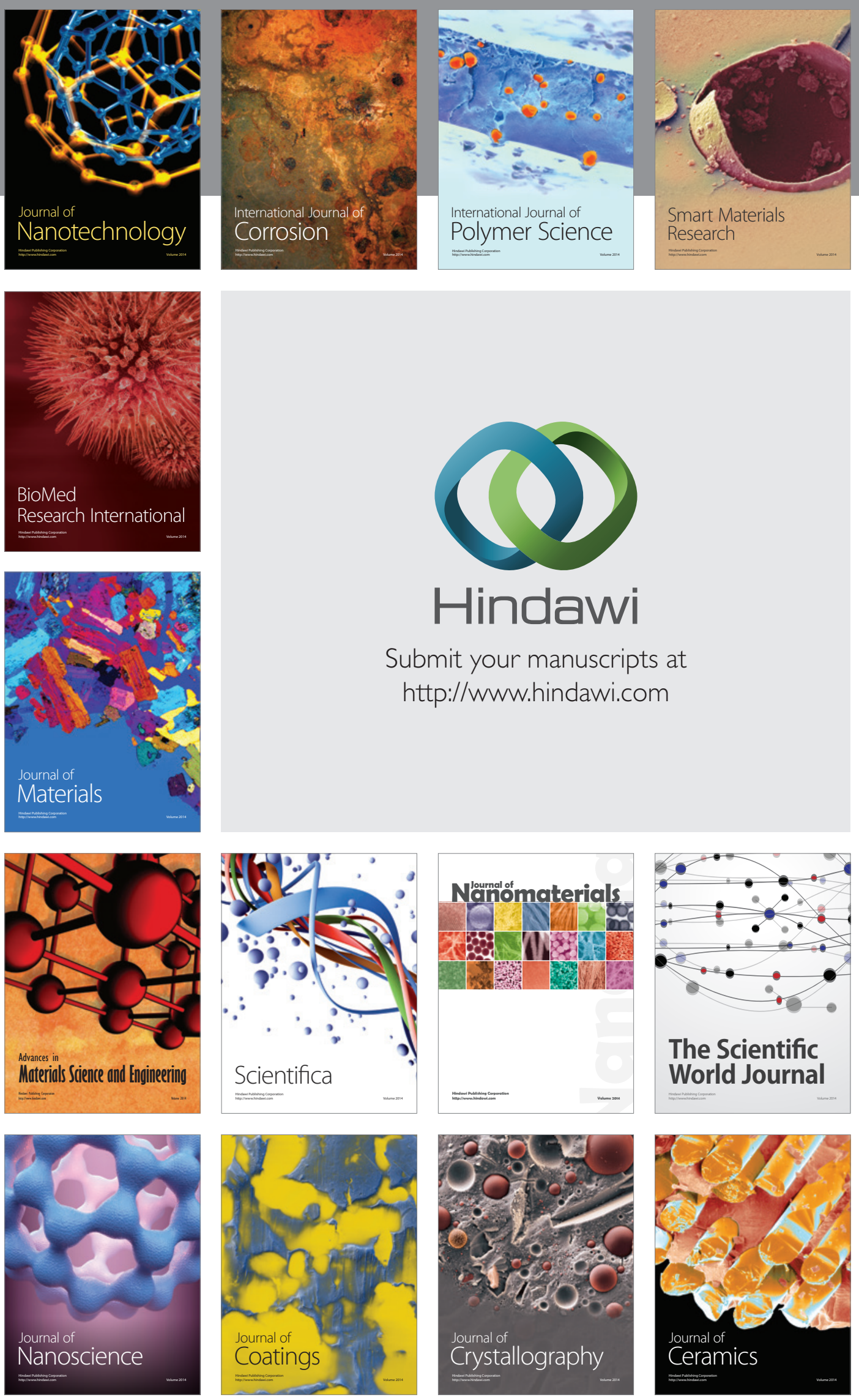

The Scientific World Journal

Submit your manuscripts at

http://www.hindawi.com

\section{World Journal}

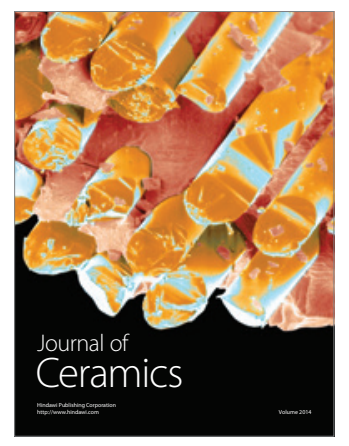

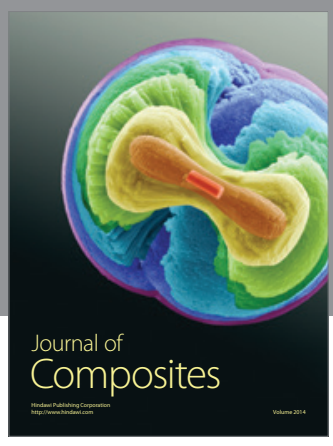
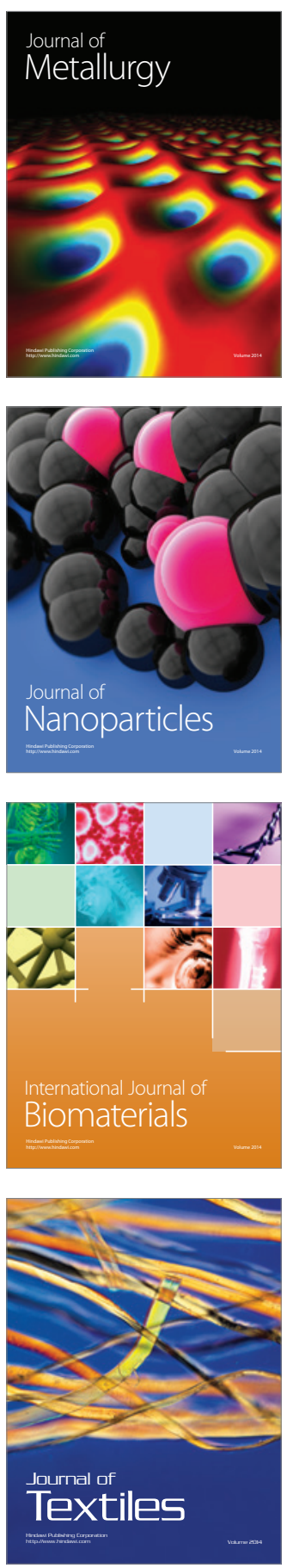